\title{
EFEITO DA DISTÂNCIA INTERLAMELAR DA PERLITA DE UM AÇO EUTETÓIDE NA DUREZA, NA SATURAÇÃO MAGNÉTICA E NO RUÍDO MAGNÉTICO DE BARKHAUUSEN*
}

\author{
Ana Paula Gonçalves Chaves ${ }^{1}$ \\ Lucas Pintol Nishikawa ${ }^{1}$ \\ Hélio Goldenstein ${ }^{1}$ \\ Linilson Rodrigues Padovese ${ }^{2}$
}

\begin{abstract}
Resumo
A pesquisa relativa ao transporte ferroviário no Brasil tem se intensificado nos últimos anos visando principalmente a melhoria no desempenho do par roda-trilho. Isso se deve a demanda de se transportar cada vez mais carga por eixo visando uma redução no custo de transporte. Através de mudanças na microestrutura do material ou de mudanças na composição das ligas utilizadas na fabricação pode se aumentar a resistência do par roda-trilho. Neste trabalho foi analisada a influência de diferentes microestruturas (puramente perlíticas e perlíticas-bainíticas), obtidas através de tratamento térmico por dilatometria, nas propriedades de um aço alto carbono similar ao utilizado em rodas convencionais classe $C$ destinadas a implementação pesada com altas cargas e baixas velocidades. Foram realizados tratamentos isotérmicos em dez temperaturas diferentes para um aço eutetóide. As análises incluíram técnicas metalográficas (definição de fases presentes e distância interlamelar da perlita), ensaios mecânicos de dureza e ensaios magnéticos (saturação magnética e ruído de Barkhausen).
\end{abstract}

Palavras-chave: Transformação de fase; Distância interlamelar da perlita; Ruído magnético de Barkhausen.

\section{THE INFLUENCE OF PEARLITE LAMELLAR DISTANCE ON HARDNESS, MAGNETIC SATURATION AND MAGNETIC BARKHAUSEN NOISE \\ Abstract}

Research about rail transport in Brazil has been intensified recently due the need to improve the wheel-rail performance, considering the demand of increasing axle load aiming for a transportation cost reduction. Changes in the material microstructure or in the alloys composition can improve the wheel-rail resistance. A eutectoid steel similar to that used in conventional class $\mathrm{C}$ wheels (designed to heavy implementation with high loads and low speeds) was submitted to isothermal heat treatments applying dilatometry techniques at ten different cycles. Different microstructures (purely pearlitic and pearlitic-bainitic) were obtained and their influence in the mechanical and magnetic properties was analyzed. The analysis included metallographic techniques (identification of phases and pearlite lamellar distance), mechanical testing and magnetic testing (magnetic saturation and magnetic Barkhausen noise).

Keywords: Phase transformation; Pearlite lamellar distance; Magnetic Barkhausen noise.

1 Escola Politécnica da USP, Departamento de Engenharia Metalúrgica e de Materiais, USP, São Paulo, SP e Brasil.

2 Escola Politécnica da USP, Departamento de Engenharia Mecânica, USP, São Paulo, SP e Brasil. 


\section{INTRODUÇÃO}

O transporte ferroviário brasileiro tem como finalidade principalmente o transporte de minérios e de grãos, implementações pesadas que demandam altas cargas transportadas por eixo à baixas velocidades. Através do aumento de carga transportada por eixo, é possível reduzir a quantidade de composições para a mesma carga transportada, reduzindo o custo. Devido as solicitações mecânicas nos materiais, é necessário melhorar o desempenho do par roda-trilho. As rodas convencionais classe $\mathrm{C}$ são utilizadas para esse tipo de implementação no Brasil, são fabricadas de aço carbono perlítico através de processos de fundição ou de forjamento. O principal mecanismo aplicado no endurecimento desses aços perlíticos é a redução do espaçamento interlamelar da perlita, que varia de acordo com a temperatura de transformação (quanto maior essa temperatura, maior será o espaçamento interlamelar). [1]

De forma a garantir a conformidade de seus produtos, a indústria ferroviária faz uso de ensaios não destrutivos. Dentre os ensaios não destrutivos destacam-se os ensaios magnéticos que, se utilizados de forma adequada, apresentam boa reprodutibilidade de resultados e baixo tempo de análise.

Um material ferromagnético quando está desmagnetizado, apresenta domínios magnéticos orientados ao acaso; quando é aplicado de um campo magnético há o crescimento dos domínios cujos momentos magnéticos estejam em orientação de menor ângulo com a direção do campo. No momento em que todos os domínios estão paralelos e apontados para a direção de magnetização ocorre a saturação magnética que é função dos momentos magnéticos presentes no material. Assim sendo, a saturação magnética é função da fração de fase ferromagnética no mesmo. [2]

Esta movimentação das paredes dos domínios magnéticos não é suave, mas sim descontínua e irregular gerando variações do campo magnético durante a magnetização. Esse movimento foi detectado por Barkhausen em 1919 utilizando um sistema de som levando o fenômeno a ser denominado como "Ruído de Barkhausen". [3]

O ensaio ruído magnético de Barkhausen (MBN - Magnetic Barkhausen Noise) tem atraído a atenção dos pesquisadores nos últimos anos devido ao fato de ser um método capaz de caracterizar diversas propriedades de um material e de ser um ensaio não destrutivo, ou seja, não danifica a amostra, tem boa precisão, é rápido e é fácil de ser realizado. [4]

Este trabalho pretende correlacionar a morfologia das fases presentes no material e suas durezas com os resultados de ruído de Barkhausen em um aço SAE 1080 totalmente perlítico.

As amostras serão tratadas em patamares isotérmicos variando desde $475^{\circ} \mathrm{C}$ até 700 ${ }^{\circ} \mathrm{C}$ com a intenção de obter estruturas perlíticas homogêneas com diferentes distâncias interlamelares.

\section{MATERIAIS E MÉTODOS}

Nesse trabalho foram utilizados corpos de prova confeccionados de aço SAE 1080 recebidos em formato de fio máquina com seção transversal de $5 \mathrm{~mm}$. A composição química do material é apresentada na Tabela 1.

Tabela 1 - Composição química em \% em peso do material utilizado

\begin{tabular}{|c|c|c|c|c|c|c|}
\hline $\mathrm{S}$ & $\mathrm{C}$ & $\mathrm{Mn}$ & $\mathrm{P}$ & $\mathrm{Si}$ & $\mathrm{Al}$ & $\mathrm{N}$ \\
\hline 0,0062 & 0,8010 & 0,5111 & 0,0073 & 0,1780 & 0,0002 & 0,0029 \\
\hline
\end{tabular}




\subsection{Ciclos Térmicos}

Os ciclos térmicos foram realizados com a utilização do dilatômetro Bähr modelo DIL805A utilizando corpos de prova cilíndricos e maciços com $4 \mathrm{~mm}$ de diâmetro e 10 $\mathrm{mm}$ de comprimento usinados do centro do arame.

Os corpos de prova foram aquecidos à uma taxa de $10^{\circ} \mathrm{C} / \mathrm{s}$ até o patamar de temperatura de $800^{\circ} \mathrm{C}$. Foram mantidos nessa temperatura por 5 minutos e resfriados com a utilização de um fluxo de gás hélio a uma taxa de $150^{\circ} \mathrm{C} / \mathrm{s}$ até os patamares isotérmicos de $700^{\circ} \mathrm{C}, 675^{\circ} \mathrm{C}, 650^{\circ} \mathrm{C}, 625^{\circ} \mathrm{C}, 600^{\circ} \mathrm{C}, 575^{\circ} \mathrm{C}, 550^{\circ} \mathrm{C}, 525^{\circ} \mathrm{C}, 500^{\circ} \mathrm{C}$ e $475^{\circ} \mathrm{C}$. Cada corpo de prova permaneceu nesse patamar de temperatura até que as transformações cessassem, por fim foram resfriadas ao ar. Cada condição de ensaio foi repetida três vezes. Na Figura 1 observa-se o desenho esquemático dos ciclos térmicos.

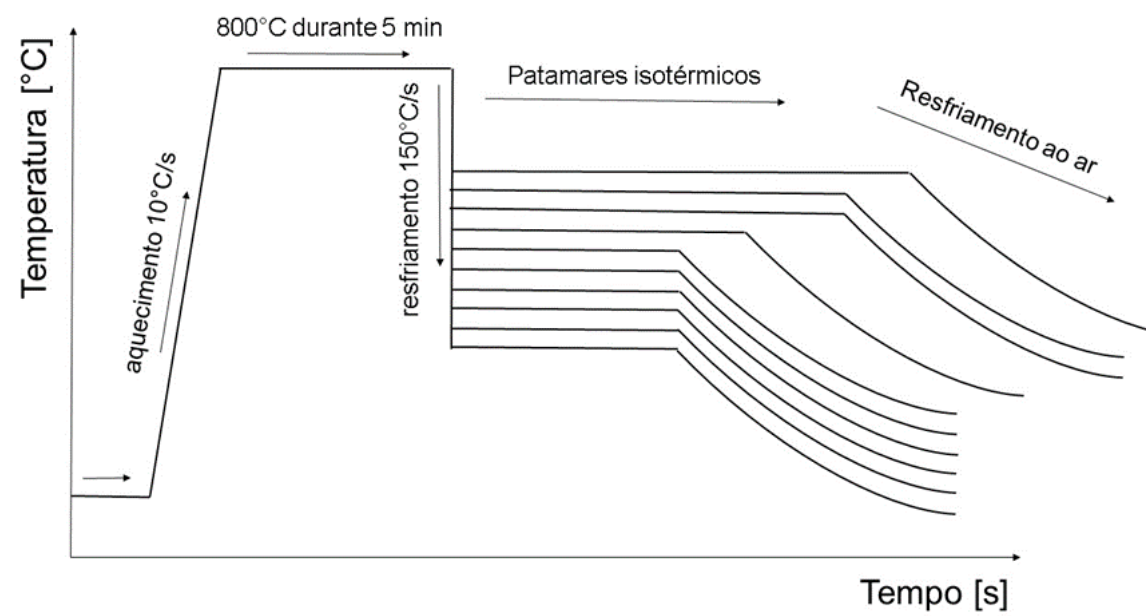

Figura 1. Desenho esquemático dos ciclos térmicos

\subsection{Análise Microestrutural e Microdureza}

Para a análise microestrutural as amostras foram lixadas, polidas com solução de diamante até $1 \mu \mathrm{m}$ e atacadas por uma solução de Nital $2 \%$. As amostras foram analisadas por microscopia ótica eletrônica de varredura de alta resolução MEV-FEG. A distância interlamelar foi determinada a partir de imagens de três diferentes regiões, para cada condição, traçando-se uma linha sobre a perlita com menor distância interlamelar e dividindo-se o tamanho total da linha pelo número de intersecções com as lamelas de cementita.

Num tratamento isotérmico, supõe-se que a distância interlamelar da perlita é constante ao longo de toda amostra, assim é válido medir a menor distância interlamelar visualizada na imagem, pois corresponde à região em que as lamelas perlíticas estão mais perpendiculares à superfície da amostra. O método utilizado para determinação da distância interlamelar média foi o método da interseção aleatória. [5] Para definição do espaçamento interlamelar mínimo, foi utilizada a Equação 1, sendo que $L_{T}$ é o comprimento total da linha de interseção traçada em $\mu \mathrm{m}$ e $I$ é o número de interseções da linha com as lamelas de cementita.

$$
S_{0 \text { mínimo }}=\frac{L_{T}}{I}(1)
$$

Para as medidas de microdureza realizaram-se 30 medidas ao longo de cada amostra utilizando-se carga de 100 gramas de acordo com norma ASTM [6] 


\subsection{Saturação Magnética e Ruído Magnético de Barkhausen}

A saturação magnética é medida em Tesla $[T]$ e seu valor pode ser obtido através da aplicação de um campo magnético elevado e fixo na amostra. Foi utilizado para análise o equipamento de saturação magnética de campo fixo. Os corpos de prova são fixados em um suporte especial e colocados entre dois imãs permanentes e envoltos por uma bobina medidora; tais imãs são fixados de forma que permaneçam o mais próximo possível da amostra. A montagem esquemática do equipamento é visualizada na Figura 2. As amostras são submetidas ao campo magnético fixo com intensidade de aproximadamente $700 \mathrm{kA} / \mathrm{m}$.

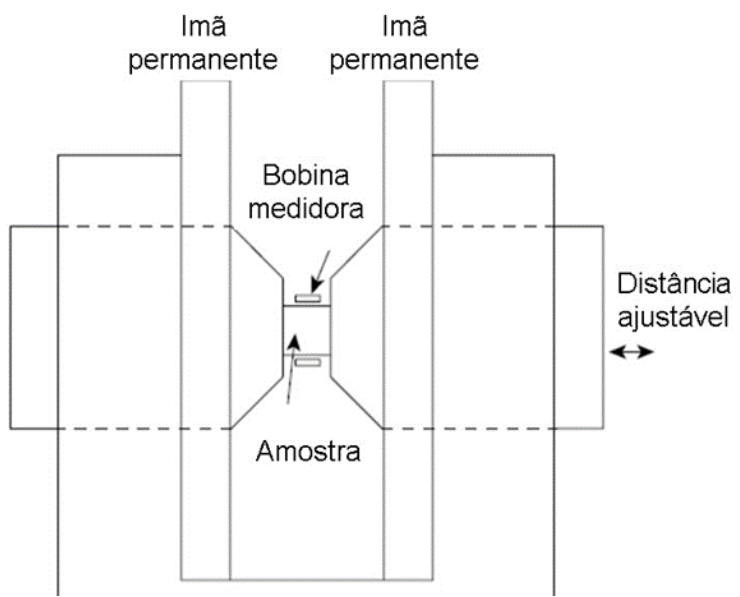

Figura 2. Desenho esquemático do equipamento de medição de saturação magnética. [3]

As amostras previamente tratadas no dilatômetro foram ensaiadas de forma a obter 0 ruído magnético de Barkhausen para cada tratamento isotérmico. A amostra foi posicionada dentro de uma bobina e submetida a magnetização cíclica. Os ensaios foram replicados para cada temperatura de tratamento isotérmico.

\section{RESULTADOS E DISCUSSÃO}

$\mathrm{Na}$ Figura 3 observam-se as imagens das microestruturas obtidas através do microscópio eletrônico de varredura. Nas imagens 3(a) e 3(b), temperaturas isotérmicas de $475^{\circ} \mathrm{C}$ e de $500^{\circ} \mathrm{C}$ respectivamente, nota-se a presença de uma mistura de perlita degenerada e de bainita superior (indicada por flechas nas figuras). Nas outras imagens, 3(c) até 3(j), observa-se uma estrutura totalmente perlítica, percebe-se também que a distância interlamelar da perlita aumenta de acordo com o acréscimo da temperatura do patamar isotérmico. 


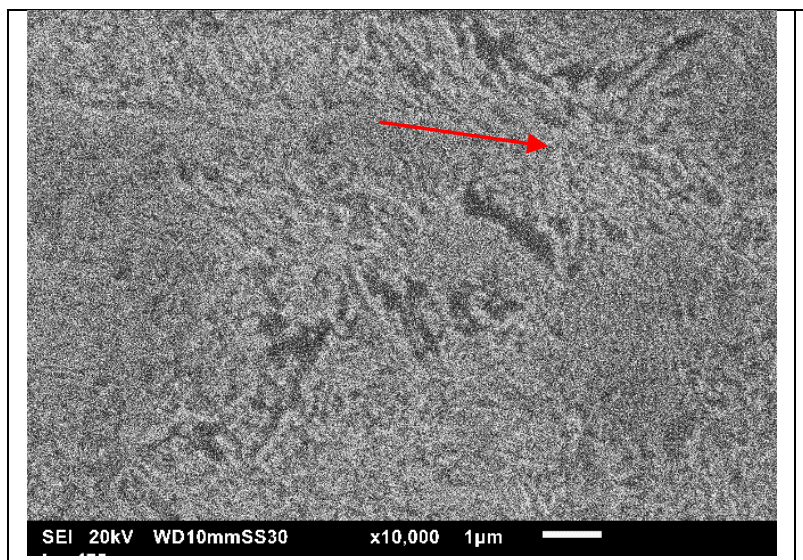

(a) Temperatura isotérmica $475^{\circ} \mathrm{C}$
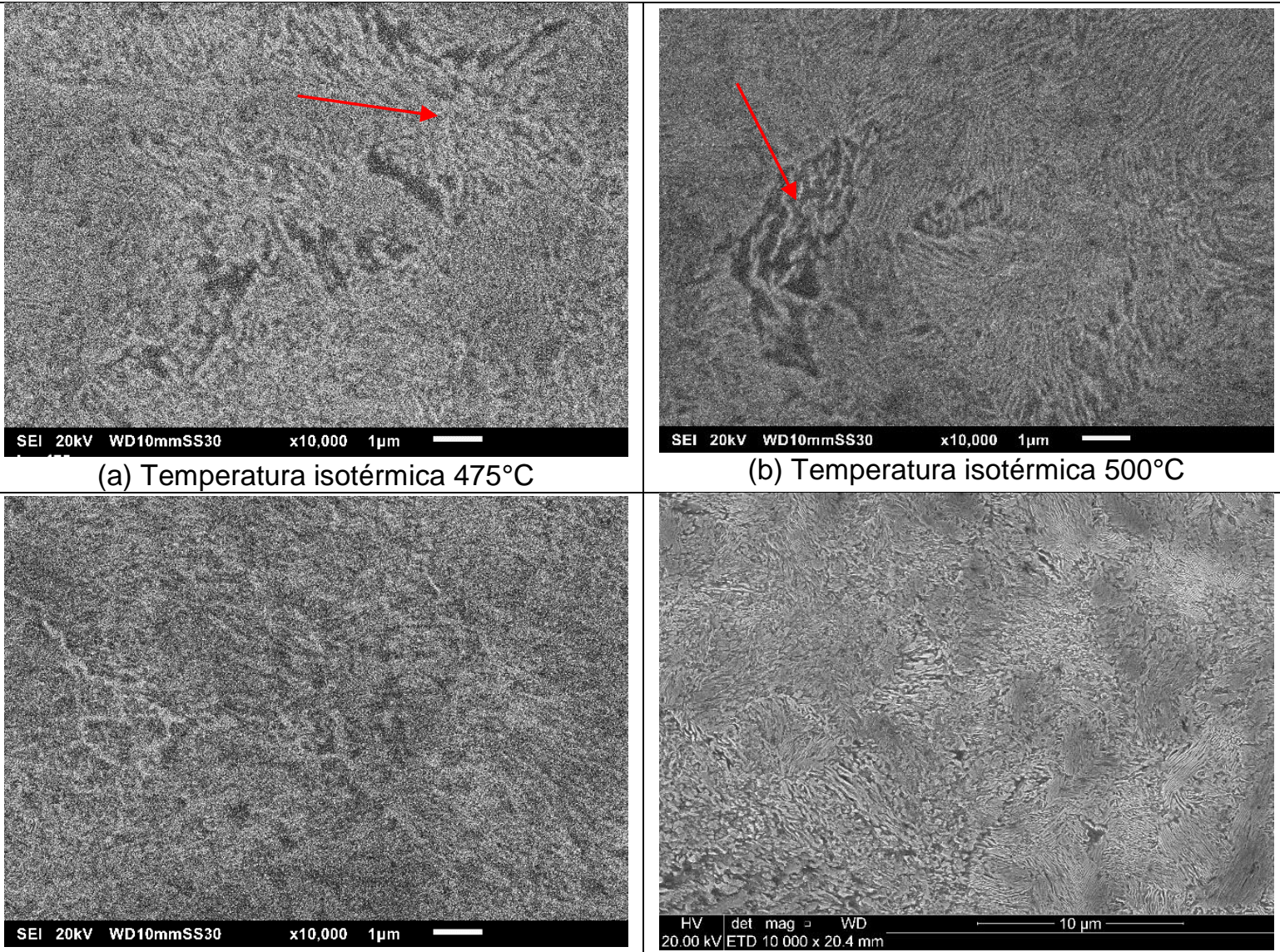

(b) Temperatura isotérmica $500^{\circ} \mathrm{C}$

(c) Temperatura isotérmica $525^{\circ} \mathrm{C}$

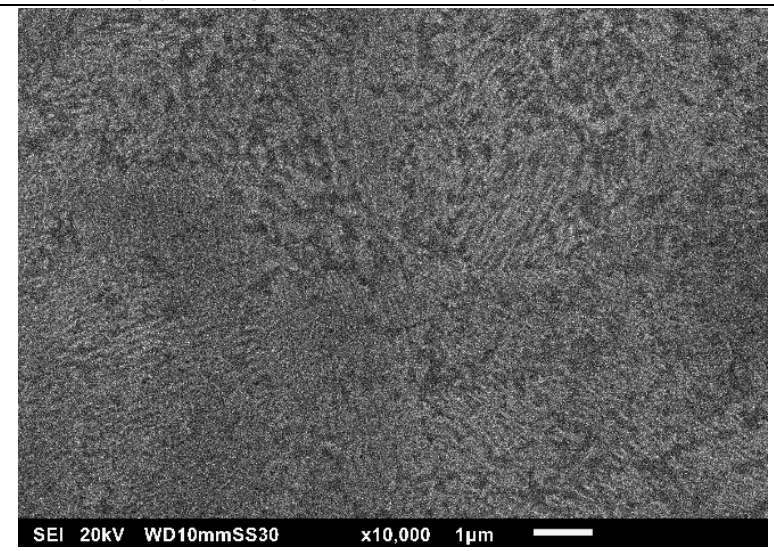

(e) Temperatura isotérmica $575^{\circ} \mathrm{C}$

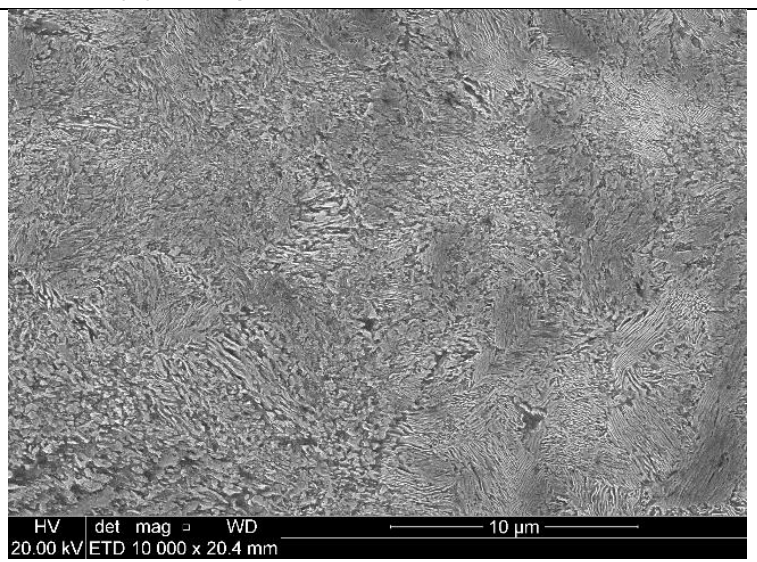

(d) Temperatura isotérmica $550^{\circ} \mathrm{C}$
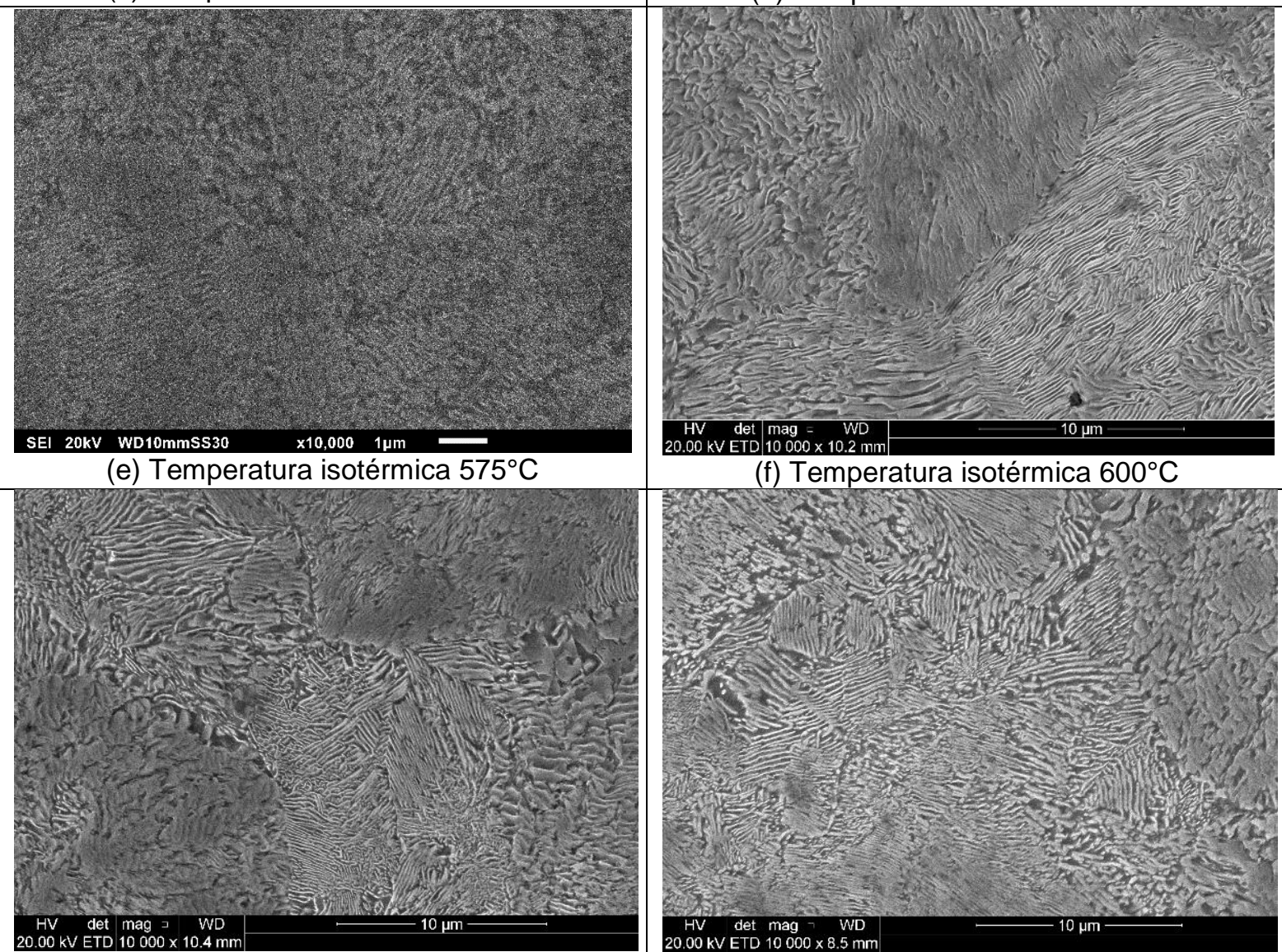

(g) Temperatura isotérmica $625^{\circ} \mathrm{C}$

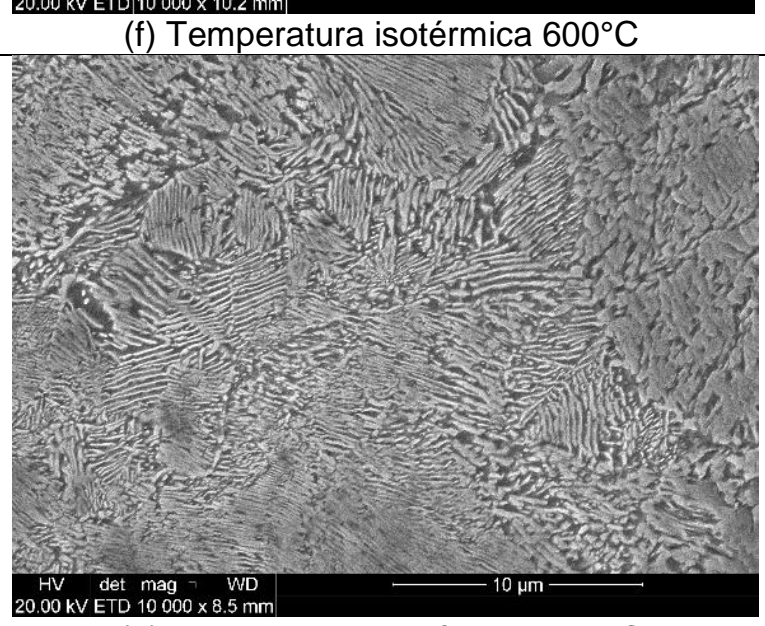

(h) Temperatura isotérmica $650^{\circ} \mathrm{C}$ 

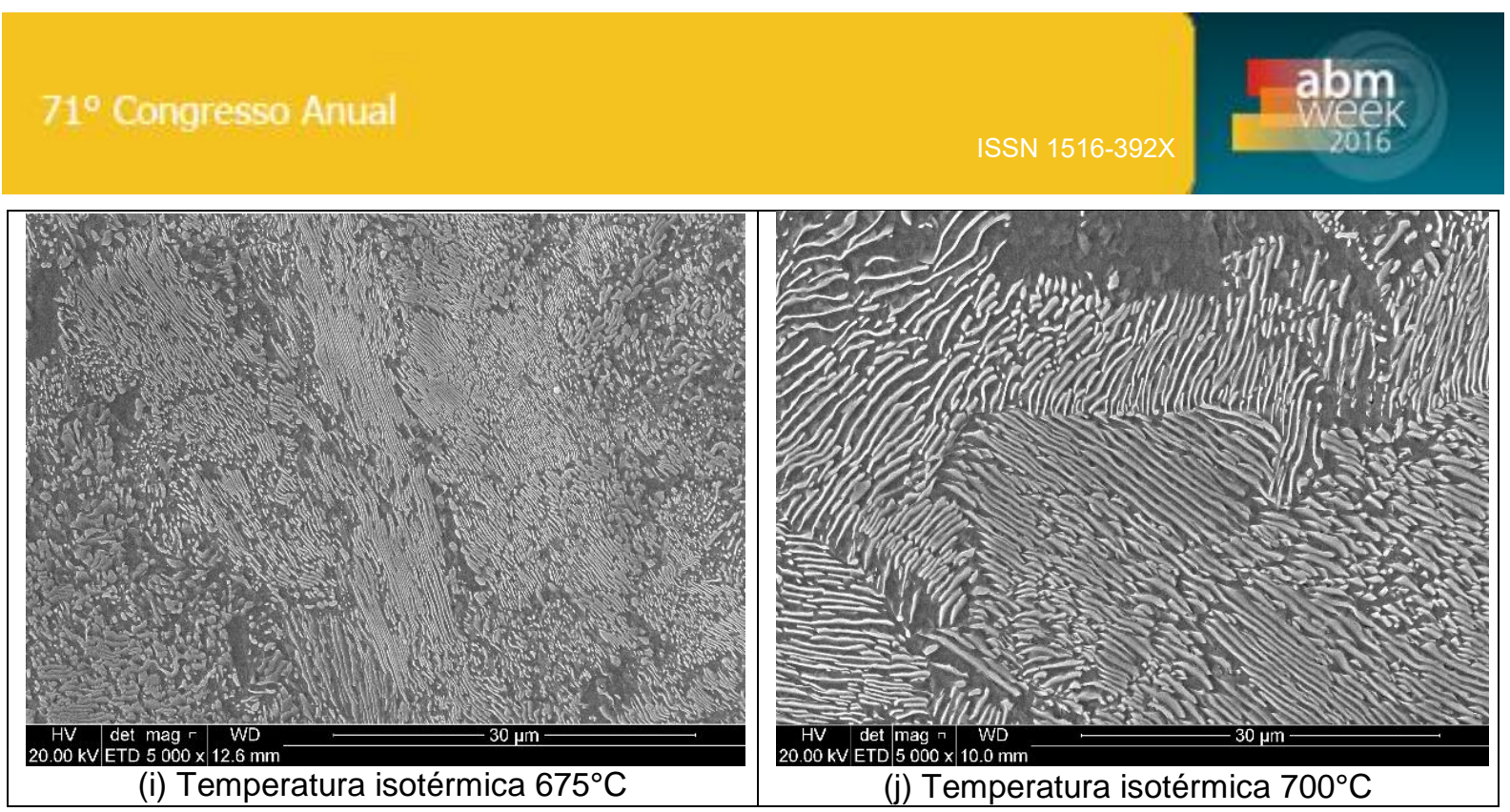

Figura 3. Microestruturas obtidas no MEV para produtos de reação isotérmica

Os valores de distância interlamelar são mostrados na Figura 4.

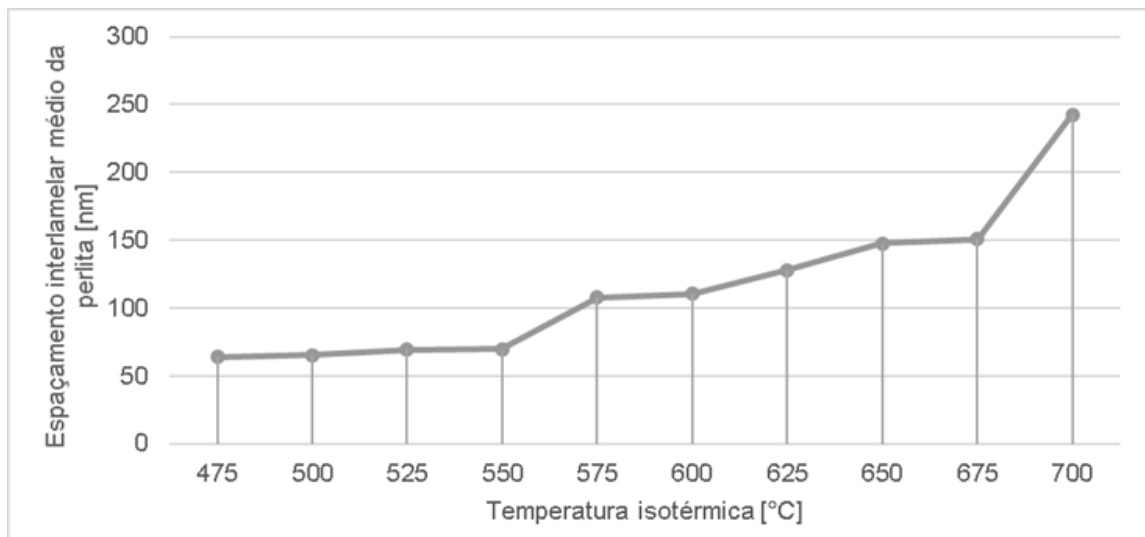

Figura 4. Espaçamento interlamelar médio em função da temperatura de transformação isotérmica.

A Figura 5 mostra a correlação do tipo Hall-Petch entre entre $1 / \sqrt{l}$ da perlita e a dureza Vickers. (Equação 2):

$$
H V=58+\frac{2824}{\sqrt{l}}(2)
$$

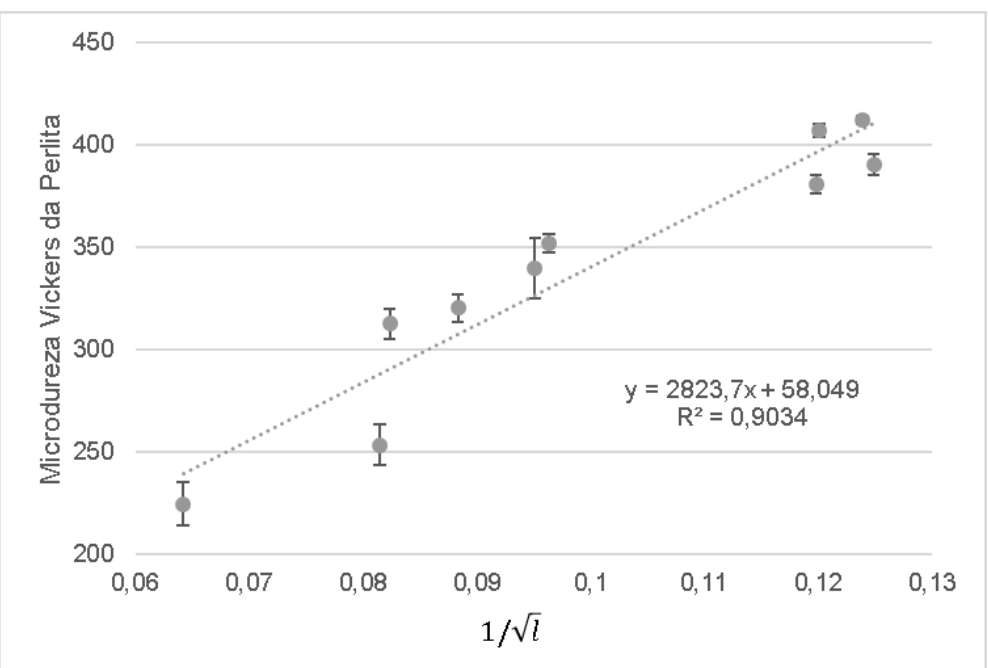

Figura 5. Correlação entre $1 / \sqrt{l}$ da perlita e a dureza Vickers 
A polarização magnética depende apenas da fração volumétrica das fases presentes, cada fase tem sua polarização característica de saturação que depende apenas da composição química e não varia de acordo com a morfologia do grão ou defeitos. [7]. Os valores de saturação magnética, observados na Figura 6 não mostram variação em função da temperatura isotérmica, indicando que a fração formada de material não magnético (cementita) é a mesma para todos os tratamentos.

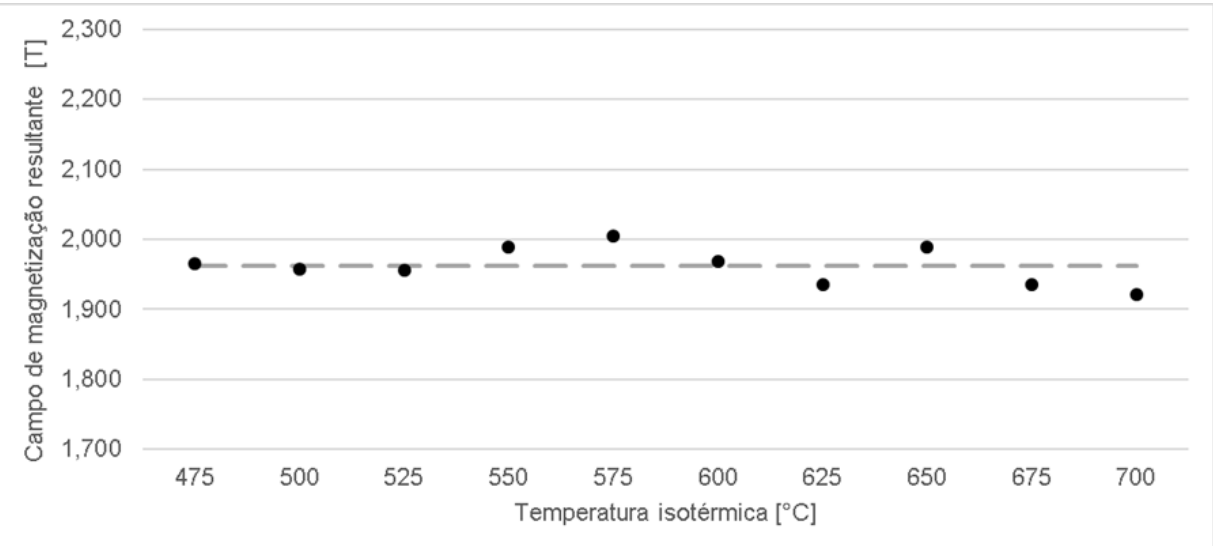

Figura 6. Campo de magnetização média em função da temperatura de transformação isotérmica

A intensidade do ruído de Barkhausen é medida pela raiz quadrada da média dos quadrados dos valores em Volt, RMS.

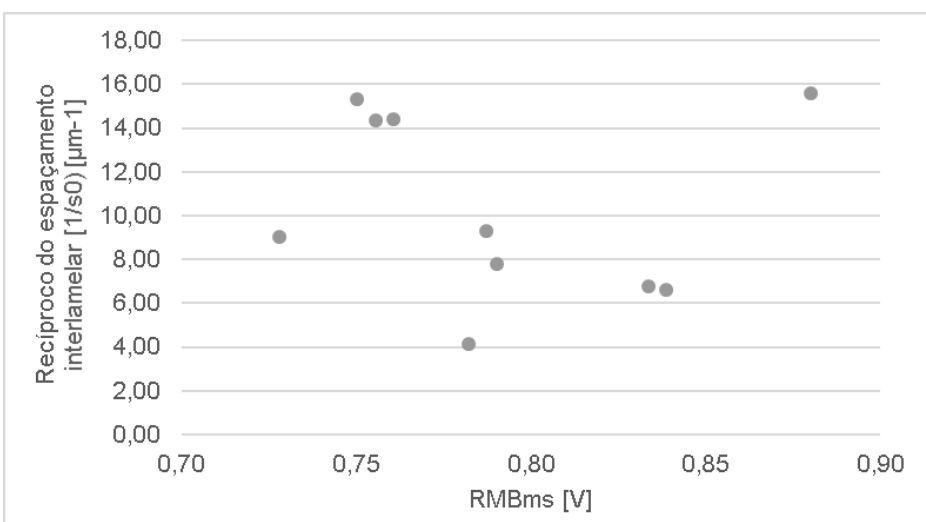

Figura 7. Recíproco do espaçamento interlamelar em função do ruído magnético de Barkhausen

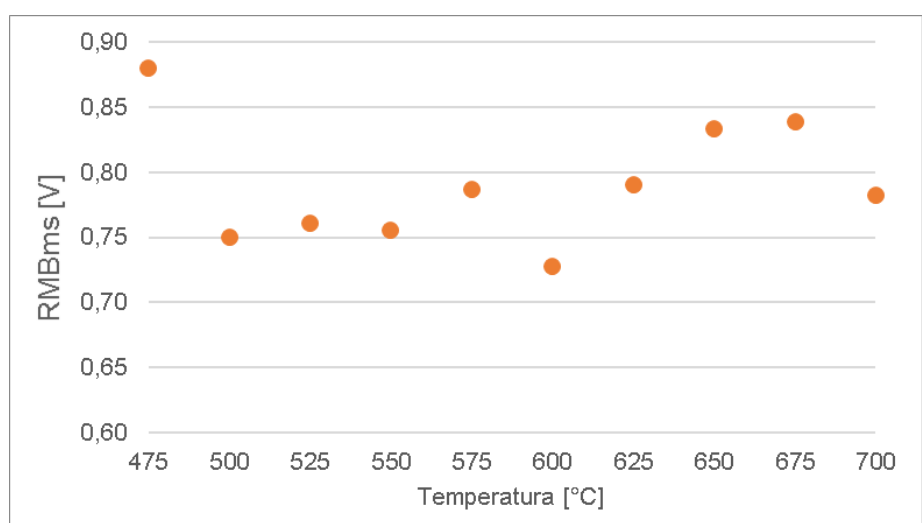

Figura 8. Ruído magnético de Barkhausen em função da temperatura de tratamento isotérmico 
Não foi possível observar uma correlação entre os valores RMS [V] com o recíproco do espaçamento interlamelar (Figura 7) nem com a temperatura de tratamento isotérmico (Figura 8). No entanto, a amostra tratada a $475^{\circ} \mathrm{C}$, que apresenta considerável quantidade de bainita superior apresenta intensidades do ruído de Barkhausen maiores do que as amostras totalmente perlíticas, sugerindo que 0 método se preste a separar os dois tipos de microconstituintes.

Segundo alguns pesquisadores, o método de ruído de Barkhausen mostra-se satisfatório para caracterizar diversas microestruturas do mesmo material (aço) que passou por diferentes tratamentos térmicos. [8] [9]

Observa-se que o ruído magnético de Barkhausen talvez não seja sensível para caracterizar diferentes espaçamentos interlamelares da perlita.

\section{CONCLUSÃO}

Este trabalho teve como objetivo analisar a relação entre a microestrutura perlítica e bainítica e as propriedades magnéticas.

Todos os tratamentos isotérmicos resultaram em estruturas lamelares, perlíticas, com exceção do tratamento isotérmico a $475^{\circ} \mathrm{C}$, que resultou em uma mistura de perlita degenerada e bainita superior, que surpreendentemente revelou-se com dureza inferior à de material de perlita fina (temperatura isotérmica de $525^{\circ} \mathrm{C}$ ).

Obteve-se a relação entre distância interlamelar, microdureza e temperatura de tratamento isotérmico. Estes dados foram relacionados com medidas de saturação magnética e ruído magnético Barkhausen.

Observou-se que os valores de saturação magnética independem da temperatura de tratamento isotérmico, indicando que a fração não magnética formada (cementita) é a mesma para todos os tratamentos.

Não se observou uma correlação entre os valores RMS [V] com o recíproco do espaçamento interlamelar da perlita. No entanto, a amostra tratada a $475^{\circ} \mathrm{C}$, que apresenta considerável quantidade de bainita superior apresenta intensidades do ruído de Barkhausen maiores do que as amostras totalmente perlíticas, sugerindo que o método se preste a separar os dois tipos de microconstituintes.

\section{Agradecimentos}

Os autores reconhecem o apoio financeiro da CAPES, CNPQ e Fundação Vale À Arcelor-Mittal, na pessoa do Eng. Helder Carvalho Ferreira, pela disponibilização do aço eutetóide utilizado neste trabalho.

Ao Laboratório de Dinâmica e Instrumentação da Escola Politécnica da USP pelo apoio nas medidas de ruído de Barkhausen.

\section{REFERÊNCIAS}

1 Zener, Kinetics of the Decomposition of Austenite. AMERICAN INSTITUTE OF MINING AND METALLURGICAL ENGINEERS, Technical Publication No. 19a5, Fall Meeting 1945/ Trans. AIME, 1946, 167:550-595

2 Faria, NR, Lima LFCP. Introdução ao magnetismo dos materiais. Editora Livraria da Física; 2005.

3 Cullity BD, Graham CD. Introduction to Mangetic Materials. $2^{\circ}$ Edição. NJ - USA: John Wiley \& Sons; 2009. 
4 Anglada-Rivera J, Padovese LR, Capó-Sanchez J. Magnetic Barkhausen noise and hysteresis loop in commercial carbon steel: influence of applied tensile stress and grain size. Journal of Magnetism and Magnetic Materials. 2001; v. 231: 299-306.

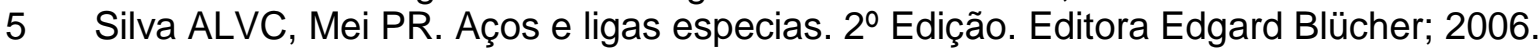

6 ASTM E384: Standard Test Method for Knoop and Vickers Hardness of Materials. 143; 2012.

7 Ramírez M F G. Estudo da transformação durante o resfriamento contínuo e da microestrutura do aço microligado $X 80$ utilizado na construção de tubos para transporte de gás natural e petróleo. Dissertação de Mestrado - USP. 2008.

8 Saquet $O$, Chicois J, Vincent, A. Barkhausen noise from plain carbon steels: analysis of the influence of microstructure. Materials Science and Engineering. 1999; vol. A269: 73-82.

9 Kameda J, Ranjan R. Nondestructive evaluation of steels using acoustic and magnetic Barhkausen signals - I Effect of carbide preciptation and hardness. Acta Metallurgica. 1987; vol.35: 1515-1526. 